\title{
Zenith angle dependence of pressure effect in GRAPES-3 muon telescope
}

\author{
M. Zuberi, ${ }^{a}{ }^{*}$ S. Ahmad ${ }^{c}$ M. Chakraborty, ${ }^{a}$ A. Chandra,${ }^{c}$ S.R. Dugad ${ }^{a}$ U.D. Goswami, ${ }^{l}$ \\ S.K. Gupta, ${ }^{a}$ B. Hariharan, ${ }^{a}$ Y. Hayashi, ${ }^{b}$ P. Jagadeesan, ${ }^{a}$ A. Jain, ${ }^{a}$ P. Jain, ${ }^{d}$ \\ S. Kawakami, ${ }^{b}$ H. Kojima, ${ }^{e}$ S. Mahapatra, ${ }^{i}$ P.K. Mohanty, ${ }^{a}$ R. Moharana,${ }^{j}$ Y. Muraki, ${ }^{g}$ \\ P.K. Nayak, ${ }^{a}$ T. Nonaka, ${ }^{h}$ A. Oshima, ${ }^{e}$ B.P. Pant, ${ }^{j}$ D. Pattanaik, ${ }^{a, i}$ G.S. Pradhan, ${ }^{k}$ \\ P.S. Rakshe, ${ }^{a}$ M. Rameez, ${ }^{a}$ K. Ramesh, ${ }^{a}$ L.V. Reddy, ${ }^{a}$ R. Sahoo, ${ }^{k}$ R. Scaria, ${ }^{k}$
} S. Shibata, ${ }^{e}$ J. Soni, ${ }^{d}$ K. Tanaka ${ }^{f}$ and F. Varsi ${ }^{d}$ [The GRAPES-3 Collaboration]

${ }^{a}$ Tata Institute of Fundamental Research, Homi Bhabha Road, Mumbai 400005, India

${ }^{b}$ Graduate School of Science, Osaka City University, Osaka 558-8585, Japan

${ }^{c}$ Aligarh Muslim University, Aligarh 202002, India

${ }^{d}$ Indian Institute of Technology Kanpur, Kanpur 208016, India

${ }^{e}$ College of Engineering, Chubu University, Kasugai, Aichi 487-8501, Japan

${ }^{f}$ Graduate School of Information Sciences, Hiroshima City University, Hiroshima 731-3194, Japan

${ }^{g}$ Institute for Space-Earth Environmental Research, Nagoya University, Nagoya 464-8601, Japan

${ }^{h}$ Institute for Cosmic Ray Research, Tokyo University, Kashiwa, Chiba 277-8582, Japan

${ }^{i}$ Utkal University, Bhubaneshwar 751004, India

${ }^{j}$ Indian Institute of Technology Jodhpur, Jodhpur 342037, India

${ }^{k}$ Indian Institute of Technology Indore, Indore 453552, India

${ }^{l}$ Dibrugarh University, Dibrugarh 786004, India

E-mail: meeran.zuberihep@gmail.com

\begin{abstract}
A large area $\left(560 \mathrm{~m}^{2}\right)$ muon telescope in the GRAPES-3 experiment at Ooty, India records muon intensity at high cutoff rigidities $\left(\mathrm{R}_{C}\right)$ varies from $14-32 \mathrm{GV}$ along 169 independent directions spanning a field of view of $2.3 \mathrm{sr}$. The threshold energy of the recorded muons is $\sec (\theta) \mathrm{GeV}$ along a direction with a zenith angle $(\theta)$ and with the average angular accuracy of $\sim 4^{o}$. The directional capabilities of the muon telescope are exploited for studying the effect of atmospheric pressure on the muon flux as a function of $\mathrm{R}_{C}$. It is observed that the barometric coefficients relationship with logarithmic $\mathrm{R}_{C}$ can be well described by second-order polynomial function with a high Spearman Rank correlation coefficient of 0.99 .
\end{abstract}

$37^{\text {th }}$ International Cosmic Ray Conference (ICRC 2021)

July 12 th - 23rd, 2021

Online - Berlin, Germany

\footnotetext{
*Presenter
} 


\section{Introduction}

The cosmic rays are the charged particles (mostly protons) that hit the Earth atmosphere almost isotropically. When a primary cosmic ray approaches the Earth, it firstly interacts with the Earth magnetic field (GMF) where it experiences the deflection depending on its rigidity. Afterwards, the primary cosmic ray continues to move towards Earth surface and interacts with the Earth atmospheric nuclei and produces the secondaries including muons and neutrons. Therefore, any change in the atmospheric conditions such as pressure or temperature can influence the observed cosmic ray flux at detector level [1,2]. These atmospheric effects on cosmic rays are known for several decades. There are several studies by various experiments for studying the effects of atmospheric pressure (barometric effect) [3,4] and temperature [1,5] on cosmic rays, which shows that these effects can produce significant modulations in the cosmic ray flux at the detector level. Hence, the correction of these effects becomes necessary for doing the precision measurements.

The barometric effect is directly related to the production, decay, or absorption of secondaries in the Earth atmosphere. The probability of these processes depends on the energy of primary particles. It is expected that a higher energy primary particle will produce the higher energy secondaries, which will be lesser affected by the changes in the atmospheric pressure. Therefore, the energy dependence of the atmospheric effect on secondaries can be utilised to study the relationship with geomagnetic cutoff rigidities of primaries, which quantifies the minimum energy required by a primary particle to reach that location. There are very few studies that explore the relationship of barometric coefficient with cutoff rigidities by using muons[6, 7]. However, these studies are limited to $\sim 25 \mathrm{GV}$ cutoff rigidities wheres, the GRAPES-3 Muon telescope directional rigidities vary from $\sim 14 \mathrm{GV}$ to $\sim 32 \mathrm{GV}$, which gives a unique advantage to study these phenomena in the higher rigidity ranges.

\section{The GRAPES-3 muon telescope (G3MT)}

The muon component of EAS plays a vital role in the field of cosmic rays astrophysics by distinguishing between $\gamma$-ray primary from charged cosmic rays particles. It allows doing the search for cosmic ray sources through $\gamma$-ray astronomy and studies on the variation of the nuclear composition of primary cosmic rays. The GRAPES-3 experiment has a $560 \mathrm{~m}^{2} \mathrm{G} 3 \mathrm{MT}$ which can give not only the information of muon content in a shower but also the directional information of individual muons. The G3MT comprises of 16 identical modules of $35 \mathrm{~m}^{2}$ sensitive area each using proportional counters (PRCs) as the basic building block. The PRC is a $6 \mathrm{~m} \mathrm{long}$, mild-steel, square pipe, with cross-sectional area of $10 \mathrm{~cm} \times 10 \mathrm{~cm}$ and a wall thickness of $2.3 \mathrm{~mm}$. Each module consists of 232 PRCs arranged in 4 layers. The alternate layers are placed in an orthogonal direction to track the muon in $2 \mathrm{D}$ plane. Two successive layers of PRCs are separated by a concrete block of $60 \times 60 \times 15 \mathrm{~cm}^{3}$. There are total 15 layers of concrete deployed from the bottom layer of PRCs which gives a total thickness of $550 \mathrm{~g} \mathrm{~cm}^{2}$ which corresponds to a threshold of $1 \mathrm{GeV}$ for vertically incident muons. These concrete blocks are arranged in an inverted pyramid shape to achieve an energy threshold of $1(\sec \theta) \mathrm{GeV}$ for muons incident on the detector at a zenith angle $\theta$ with coverage up to $45^{\circ}$. The muon angle data is grouped into the $13 \times 13=169$ out of 225 bins 
restricting the maximum zenith angle up to $50^{\circ}$ which still exceeds the shielding coverage of $45^{\circ}$ [8] as shown in Fig. 2.

The unique capability of directional reconstruction of the G3MT also permits exploring various transient phenomena like geomagnetic storms $[9,10]$, acceleration of particles during thunderstorms [11], etc. The effective cutoff rigidities in 169 directions are numerically calculated by using the back-tracing algorithm [12] as shown in Fig. 2. The details of the cutoff rigidity calculation can be found elsewhere [13].

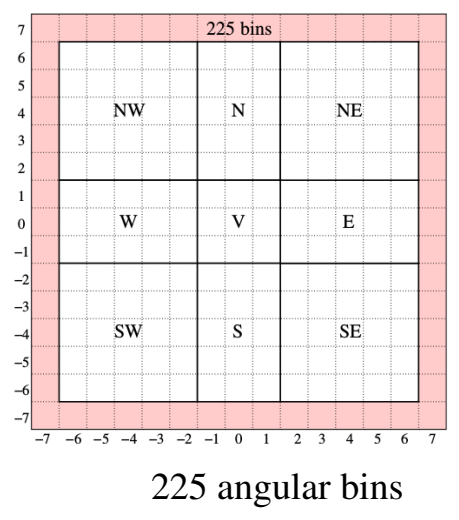

225 angular bins

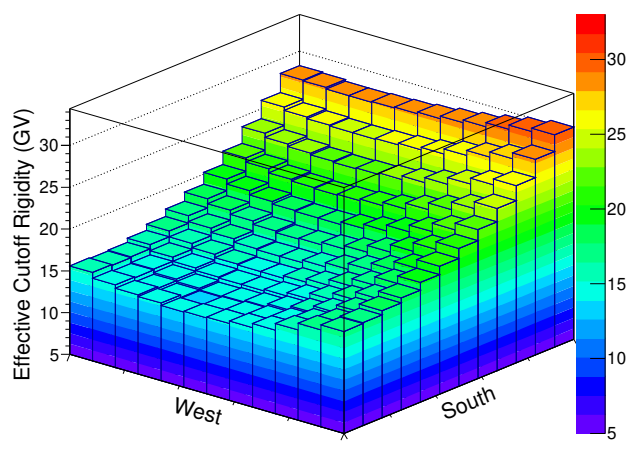

$\mathrm{R}_{C}$ variation in 2-D space

\section{Barometric coefficient calculation}

The modulation in the atmospheric pressure changes the mass of the air column above the detector, which causes the variation in secondary cosmic ray flux. However, the measured flux variation is also coupled with various solar phenomena, which needs to be isolated before studying the barometric effect. The Fast Fourier Transform (FFT) technique is used to segregate both these effects [14]. The power spectrum of (A) muon angle data for a particular direction 083, and (B) atmospheric pressure is shown in Fig.1 for combined six years (2001-2006) of data.

Both the data sets show a dominant peak at 2 cycles per day (cpd), which is expected due to the pressure effect. We have designed a bandpass filter to select the frequency centered at $2 \mathrm{cpd}$ from both the data sets and then performed Inverse Fast Fourier Transform (IFFT) on the filtered frequency components to convert them back into the time-series data. The \% change in filtered muon data is plotted against the change in filtered atmospheric pressure from its mean value, which shows a very strong anti-correlation pattern for all 169 directions. We have used the linear regression technique to determine the barometric coefficient $\left(\beta_{P}\right)$ for all 169 directions by using the following equation:

$$
\frac{\Delta I}{<I>}(\%)=\beta_{P}(\mathrm{P}-<\mathrm{P}>)
$$

Fig. 2 shows the variation for a particular direction 083 . The $\beta_{P}$ is found to be $-0.18 \% / \mathrm{hPa}$ with a high value of Pearson correlation coefficient of 0.99 .

Fig. 3 shows the variation of $\beta_{P}$ for all 169 directions in 2-D space. It is evident from the Fig.3 that the value of $\beta_{P}$ is more in the western direction as compared to the eastern ones. 


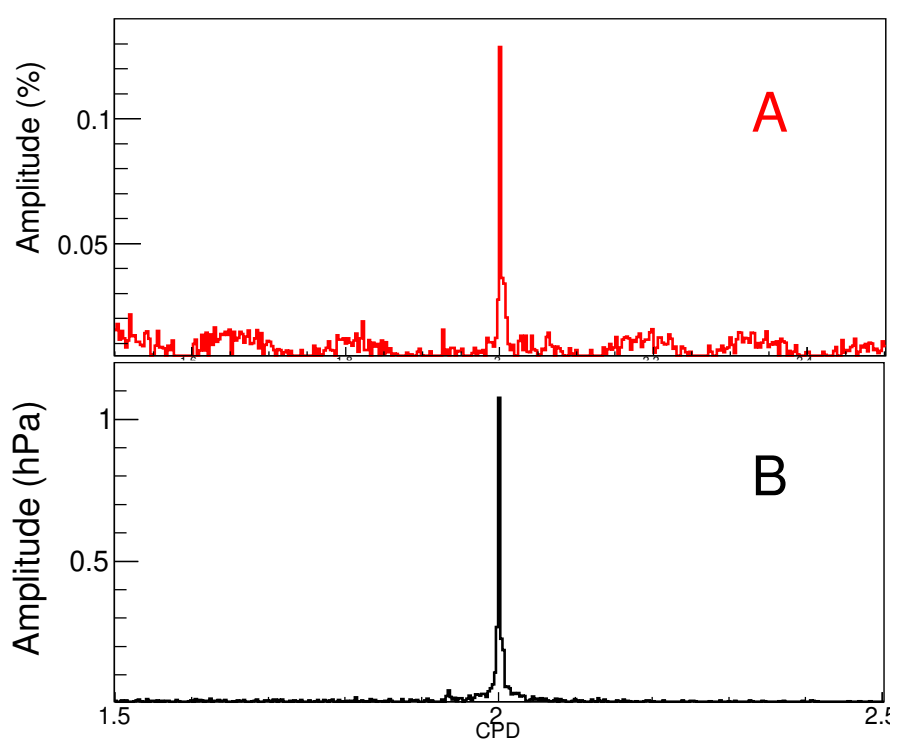

Figure 1: Power spectrum of (A) muon angle, and (B) atmospheric pressure

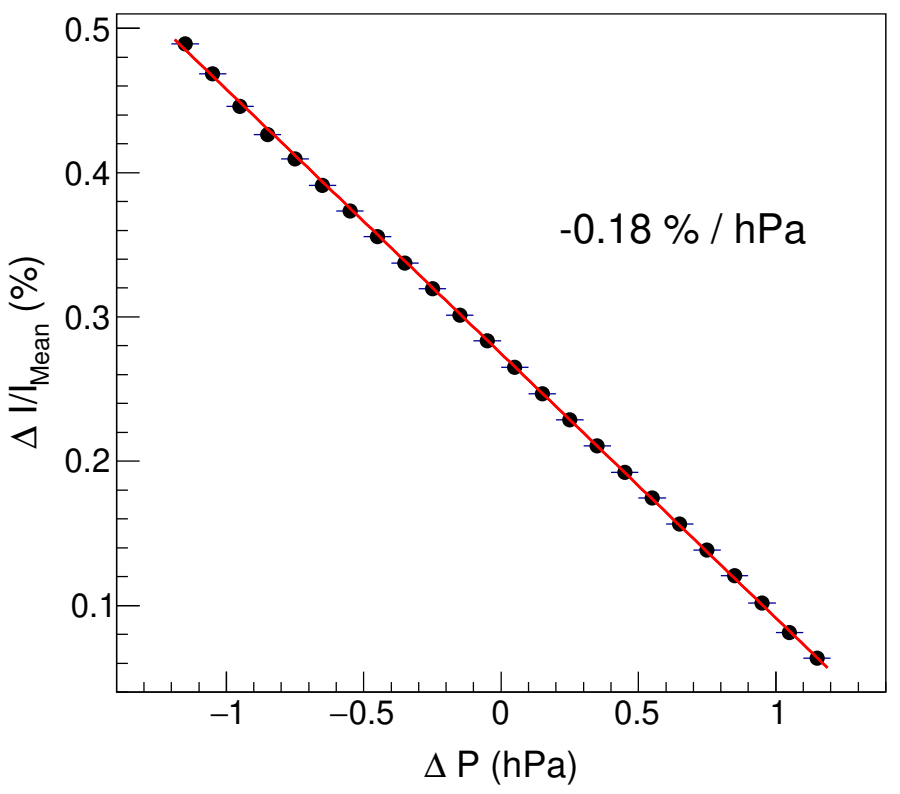

Figure 2: Change in muon flux (\%) vs atmospheric pressure

\section{Barometric coefficient dependence on $\mathbf{R}_{C}$}

The east-west asymmetry observed in $\beta_{P}$ resembles that seen in the case of $\mathrm{R}_{C}$. The Fig.4 shows the variation of $\beta_{P}$ as a function of natural logarithmic of $\mathrm{R}_{C}$.

It is observed that the value of $\beta_{P}$ decreases linearly with an increase in $\ln \left(\mathrm{R}_{C}\right)$ up to $\mathrm{R}_{C}$ $\sim 25 \mathrm{GV}$, beyond which the curve seems to get flatten. Both these features can be well modeled 


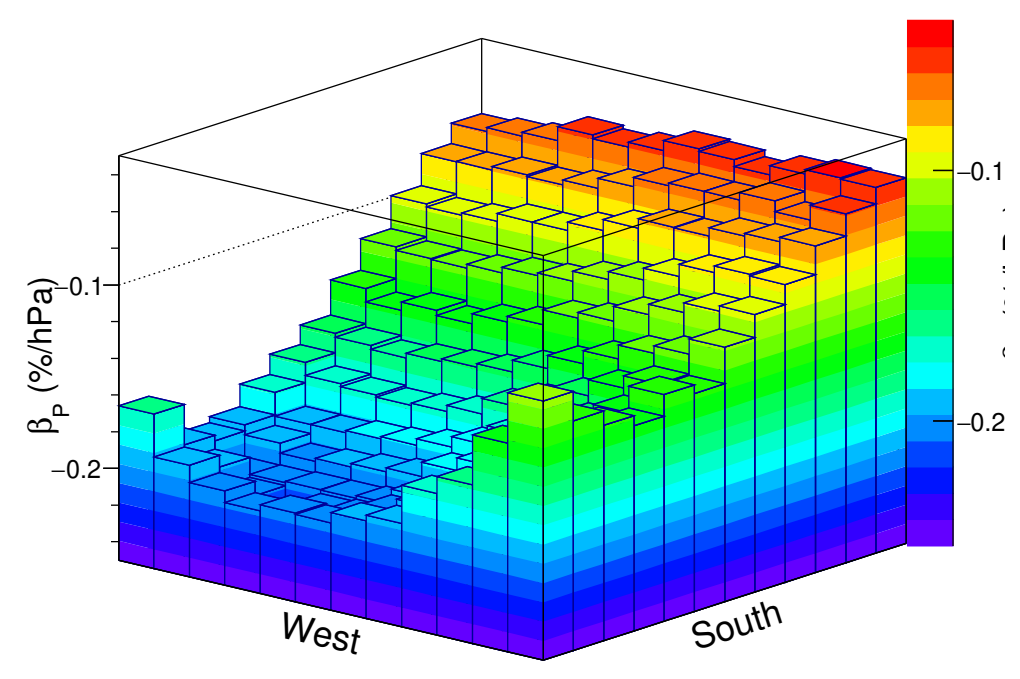

Figure 3: Variation of $\beta_{P}$ for all 169 directions in 2-D space

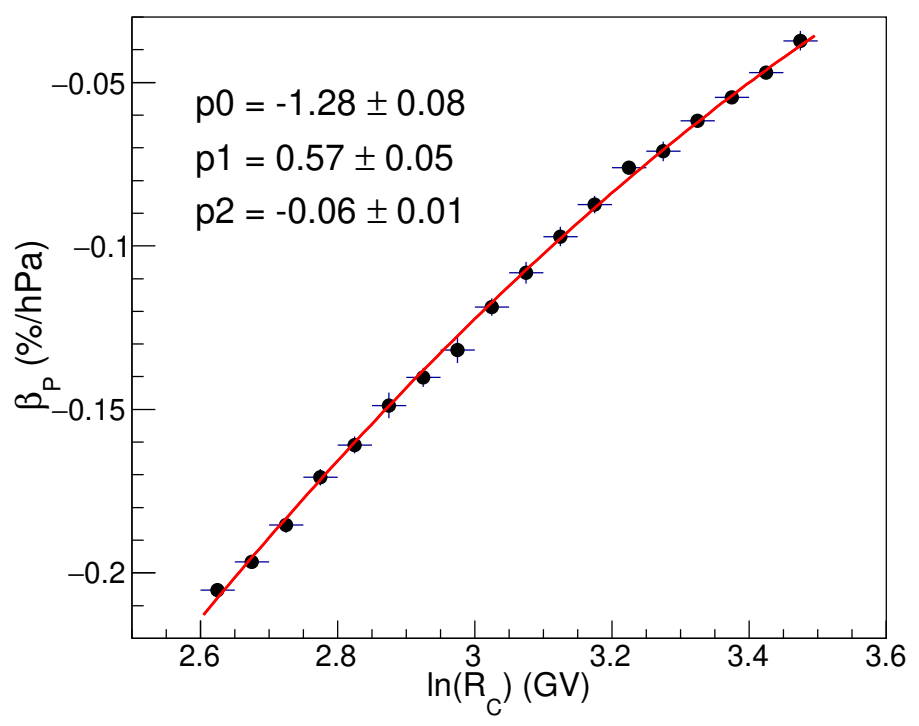

Figure 4: Variation of $\beta_{P}$ as a function of $\ln \left(\mathrm{R}_{C}\right)$

by a second-order polynomial fit, which yields the coefficient values of $\mathrm{p} 0=-1.28 \pm 0.08, \mathrm{p} 1=$ $0.57 \pm 0.05$, and $\mathrm{p} 2=-0.06 \pm 0.01$. The Spearman Rank correlation coefficient value is found to be 0.99 , which shows the tight relationship between these two variables.

\section{Conclusions}

In this study, we observed a strong correlation between muon and the atmospheric pressure in all 169 viewing directions of G3MT. The barometric coefficients are obtained with the aid of the FFT 
technique. The large cutoff rigidity variation ( $14 \mathrm{GV}$ to $32 \mathrm{GV}$ ) distributed among 169 directions are exploited to study the rigidity dependence of barometric coefficients. The coefficients show a tight relationship with the natural logarithmic of rigidities with a high Spearman Rank correlation coefficient of 0.99. The barometric coefficient was expected to show a linear relationship with $\ln \left(\mathrm{R}_{C}\right)$ but a clear departure from the linear trend can be seen at $\mathrm{R}_{C} \sim 25 \mathrm{GV}$, which can be fitted well with the second-order polynomial. However, to understand the possible physical mechanisms responsible for this peculiar behaviour of $\beta_{P}$ further work is needed.

\section{Acknowledgements}

We thank D.B. Arjunan, K. Manjunath, S. Murugapandian, S. Pandurangan, B. Rajesh, M.S. Shareef, C. Shobana, R. Sureshkumar, and other colleagues for their help in running and maintenance of the GRAPES-3 experiment.

\section{References}

[1] Dorman, L. I., Astrophysics and Space Science Library. Netherlands, Dordrecht: Springer (2004)

[2] Sagisaka, S., Il Nuovo Cimento C, 9(4), 809-828 (1986)

[3] Ambrosio, M., et al., Astroparticle Phys., 7(1-2), 109-124 (1997).

[4] Adamson, P., et al., Phys. Rev., D(81), 012001, (2010).

[5] Arunbabu, K. P., Astroparticle Physics 94, 22-28 (2017).

[6] Mendonca RRS et al., J Geophys Res: Space Phys 124(12) 9791-9813 (2019)

[7] Maghrabi et al., Advances in Astronomy, vol. https://doi.org/10.1155/2016/9620189 (2016)

[8] Y. Hayashi et al., Nucl. Instrum. Methods A 545, 643 (2005).

[9] P.K. Mohanty et al., Phys. Rev. Lett. 117, 171101 (2016).

[10] P.K. Mohanty et al., Phys. Rev. D 97, 082001 (2018).

[11] B. Hariharan et al., Phys. Rev. Lett. 122, 105101 (2019).

[12] Smart, D.F., Shea, M.A., Adv.Space Res.36, 2012 (2005)

[13] B. Hariharan et al., Experimental Astronomy 48, 111-120 (2019)

[14] P.K. Mohanty et al., Astropart. Phys. 79, 23-30 (2016). 